\title{
Epileptic Seizure Detection using Simulated Annealing based Optimal Feature Subset Selection with Kernel Extreme Learning Machine Classification Model
}

\author{
P. Suguna ${ }^{1}$, B. Kirubagari ${ }^{2}$, R. Umamaheswari ${ }^{3}$ \\ ${ }^{1}$ Research Scholar, Department of Computer Science, Annamalai University, sugunasaravanan8@gmail.com \\ ${ }^{2}$ Associate Professor, Department of Computer Science and Engineering, Annamalai University, \\ kirubathiyagu1977@gmail.com \\ ${ }^{3}$ Professor and Head, Department of Computer Science and Engineering, Gnanamani College of Engineering and \\ Technology, Namakkal, umait1978@gmail.com
}

\begin{abstract}
Electroencephalogram (EEG) signal based epileptic seizure detection is a hot research area, which identifies the non-stationary progresses of brain actions. Typically, the epilepsy is detected by doctors based on the visual examination of EEG signals consumes more time and highly sensitive to noise. Presently, machine learning (ML) techniques finds useful to predict the existence of epileptic seizure from EEG signals. This paper aims to develop a ML based Epileptic seizure detection model in EEG signals. The proposed model involves three major processes namely preprocessing, feature selection and classification. Initially, the EEG signal undergoes preprocessing in two ways namely data normalization, and class labeling. Next, simulated annealing (SA) is applied as a feature selector to choose an optimal set of features. At last, kernel extreme learning machine (KELM) based classification process takes place to detect and classify the existence of EEG. A detailed simulation analysis takes place to inspect the performance of the SA-KELM model. The experimental outcome stated that the SA-KELM model has offered maximum detection performance under the classification of multiple classes with the maximum sensitivity of $57.27 \%$, specificity of $89.32 \%$, accuracy of $82.91 \%$, F-score of $57.29 \%$ and kappa of $46.62 \%$.
\end{abstract}

Key words: Machine learning, Epileptic seizure, Classification, Feature selection, Simulated annealing

\section{INTRODUCTION}

Epilepsy is a kind of neurological disorder, which is defined by seizures affecting human beings at all ages. Epilepsy is a third most common brain infection. Based on the survey, world health organization (WHO) have reported that around 40 million peoples were affected by epilepsy. Also, it is caused due to molecular mutation, which is one reason among various causes that concludes in uneven neuronal hierarchy or movement of neurons. Though the major cause of epilepsy is still in a dark side, primary analysis is highly essential and so the treatment can be provided accordingly. Here, epilepsy affected patients are treated with some medications or surgical principles. But these models are not highly significant and effective. Early diagnosis of epileptic seizures consumes maximum time and it is applicable to prevent the disorder before reaching the critical stage. Hence, an automated tool is suitable for predicting a seizure and notifies the physician for immediate treatment to be recovered from epilepsy.

Recently, Electroencephalography (EEG) signal is applied as a major tool utilized for epilepsy prediction. The EEG signal captures the electrical brain activities [1] in an effective manner. In general, EEG is considered as a remarkable device used for epilepsy analysis and it is a cheaper machine for both long as well as short term patient's observation using any suspected epileptic syndrome [2]. The performance of EEG is based on the estimation of brain's electrical action. These actions are recorded in a system with the application of tiny metal electrodes which is fixed on the scalp. These metal electrodes are placed on the scalp while invoking the treatment and record the electrical activities of a brain. A smart method is employed for placing an electrode on the scalp. Under the application of these electrodes, signals which are obtained from the scalp are forwarded to a personal computer (PC) which is recorded with high security.

Typically, the physicians estimate the EEG values manually which is a time-consuming operation and it raises the complication of the method [3]. Therefore, a study based on the automated diagnosis of epileptic seizure is highly applicable for doctors to understand EEG values which depict a good clinical importance [4]. In last decades, numerous supervised models were deployed for the purpose of epileptic seizure analysis. Only few traditional Machine Learning (ML) 
models applied in epilepsy diagnosis are Wavelet Neural Network (WNN), random forest (RF) Support Vector Machine (SVM), Random Forest (RF), and so forth.

[5] presented an integration of ANN, Wavelet Transform (WT), as well as fuzzy relations for classifying epileptic EEG signals. [6] projected a hybrid scheme on the basis of concatenating Multi-Scale Radial Basis Function (MRBF) system and Fisher Vector (FV) encoding for the purpose of analyzing seizure epileptic. Followed by [7], the Hilbert vibration decomposition was applied for degrading EEG signals and employed Least Squares-SVM (LS-SVM) classification technology. [8] deployed an ensemble empirical mode decomposition with adaptive noise (CEEMDAN) for the classification of epileptic seizure from EEG signals, and accomplished best final outcome.

Then, [9] have developed a hybrid approach by the integration of Discrete Wavelet Transform (DWT) as well as Arithmetic Coding for classifying the epileptic seizure signals. [10] related different feature extraction, Feature Selection (FS), and classifier approaches for the prediction of epileptic signals. [11] applied a hybrid framework by merging statistical as well as spectral features of max normalized intrinsic modes for predicting EEG epileptic seizure. In conventional studies; the developers have applied heterogeneous classifiers as well as features for the classification of epileptic seizures. There are 2 various complexities experienced by developers in previous works that requires manual diagnosis and reach a simulation outcome in epileptic seizure prediction [12].

This paper develops a machine learning (ML) based Epileptic seizure detection using simulated annealing (SA) based feature selection and kernel extreme learning machine (KELM) based classification called SA-KELM model. The proposed model involves three major processes namely preprocessing, feature selection and classification. Initially, the EEG signal undergoes preprocessing in two ways namely data normalization, and class labeling. Subsequent to that, SA is applied as a feature selector to choose an optimal set of features. Finally, KELM based classification process takes place to detect and classify the existence of EEG. A detailed simulation analysis takes place to examine the classifier outcome of the proposed SA-KELM model. The performance of the SA-KELM model is tested using EEG signal dataset and a detailed comparative analysis takes place to ensure the performance of the SA-KELM model.

\section{THE PROPOSED SA-KELM MODEL}

The basic principle behind the working procedure of SA-KELM model involves preprocessing, feature extraction and classification, as shown in Figure 1. At the initial stage, preprocessing is carried out on the EEG signals in two levels namely data normalization and class labeling. Next, SA is applied as a feature extractor to select an optimal set of features. Finally, KELM is employed as a classification model to detect the existence of different classes of epileptic seizure.

\subsection{Preprocessing}

Initially, minimum-maximum (min-max) technique is utilized to normalize the dataset [13]. Here, the larger and smaller values in the collection of data are considered. Every data undergo normalization to these values. The intention is the normalization of the minimal value to 0 and maximum value to 1 , and distributes other values into the range of 0 to 1 . Eq. (1) is used to define the process of min.-max normalization.

$$
\text { Min }- \text { Max.Norm }=\frac{x-x_{\min }}{x_{\max }-x_{\min }}
$$

Finally, class labeling process takes place where the instances in the EEG signal dataset are allocated to proper class labels as 0,1 for binary class and $0,1,2,3,4$ for multi-class.

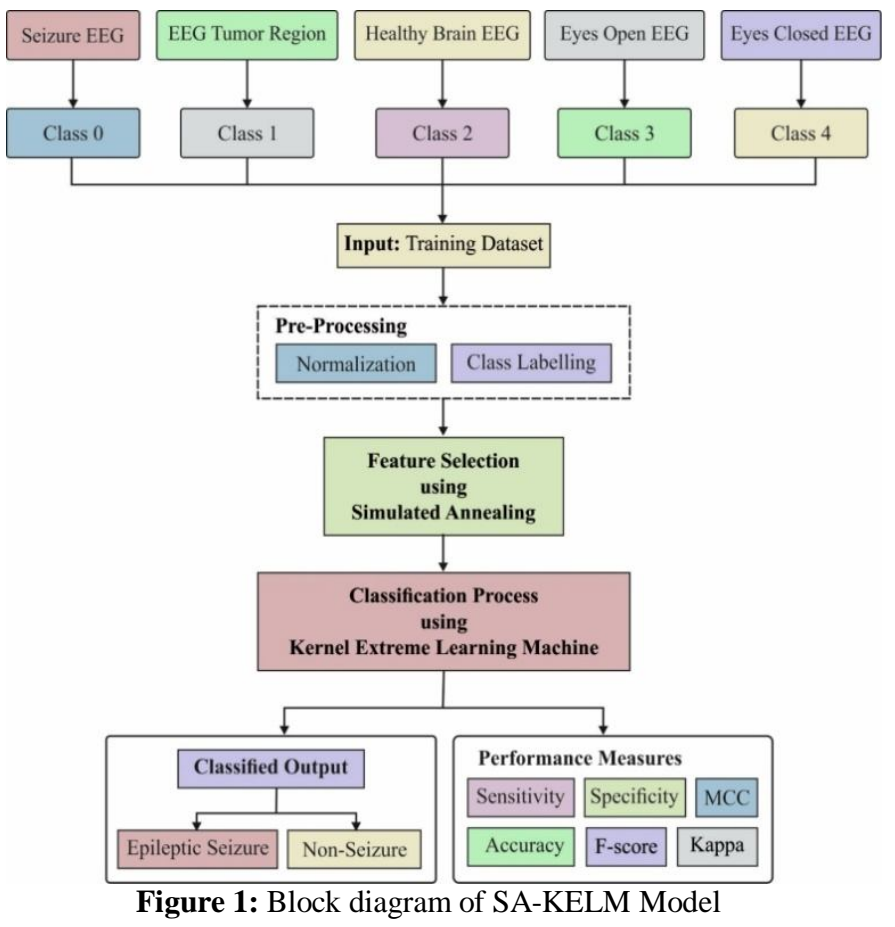

\subsection{SA based Feature Selection Process}

SA is defined as stochastic method applied on statistical models for identifying globally optimal solutions in case of huge optimization issues [14]. Followed by, SA is said to be vulnerable approach which does not require the structural details of search space. It is operated by considering few portions of the present solution which belongs to best one, and such portions have to be sustained by searching neighbors of recent solution. By considering the objective function which has to be reduced, SA moves from hill to hill and leaves the sub $\square$ optimal solutions.

If a system $S$ (set of possible states) is present in thermal equilibrium (at a given temperature $T^{\gamma}$ ), the possibility which 
is involved in specific state $s$, named as $P_{T}(s)$.It is based on $T$ and the energy $E(s)$ of state iss. The probability applies a Boltzmann distribution and is defined below.

$$
P_{T}(s)=\frac{\exp \left(-\frac{E(s)}{k T}\right)}{Z}, \text { with } Z=\sum_{s \in S} \exp \left(-\frac{E(s)}{k T}\right)
$$

where $k$ refers the Boltzmann constant as well as $Z$ is treated as a normalizing factor. Metropolis and team developed a stochastic relaxation model which operates by simulating the nature of a system at specific temperature $T$. Being $s$ the present state and $s^{\prime}$ a neighboring state, the possibility of developing a transition from $s$ to $s^{\prime}$ is defined as a ratio $P_{T}\left(s \rightarrow s^{\prime}\right)$ among the viability of being in $s$ and probability of being in $s^{\prime}$ :

$$
P_{T}\left(s \rightarrow s^{\prime}\right)=\frac{P_{T}\left(s^{\prime}\right)}{P_{T}(s)}=\exp \left(-\frac{\Delta E}{k T}\right)
$$

where it is defined that $\Delta E=E\left(s^{\prime}\right)-E(s)$. Hence, the approval or elimination of $s^{\prime}$ as a novel state is based on the variations of energies of states at temperature $T$. When $P_{T}\left(s^{\prime}\right) \geq P_{T}(s)$ and "move" is often approved. It $P_{T}\left(s^{\prime}\right)<$ $P_{T}(s)$ then it is ensured with probability $P_{T}\left(s, s^{\prime}\right)<1$.It is apparent that, the probability is based on the present temperature $T$ and reduced as $T$ as well. Finally, it is invoked with a measure of $T$ lower enough (the freezing point), whereas the transitions might be unlikely and system is assumed as frozen.

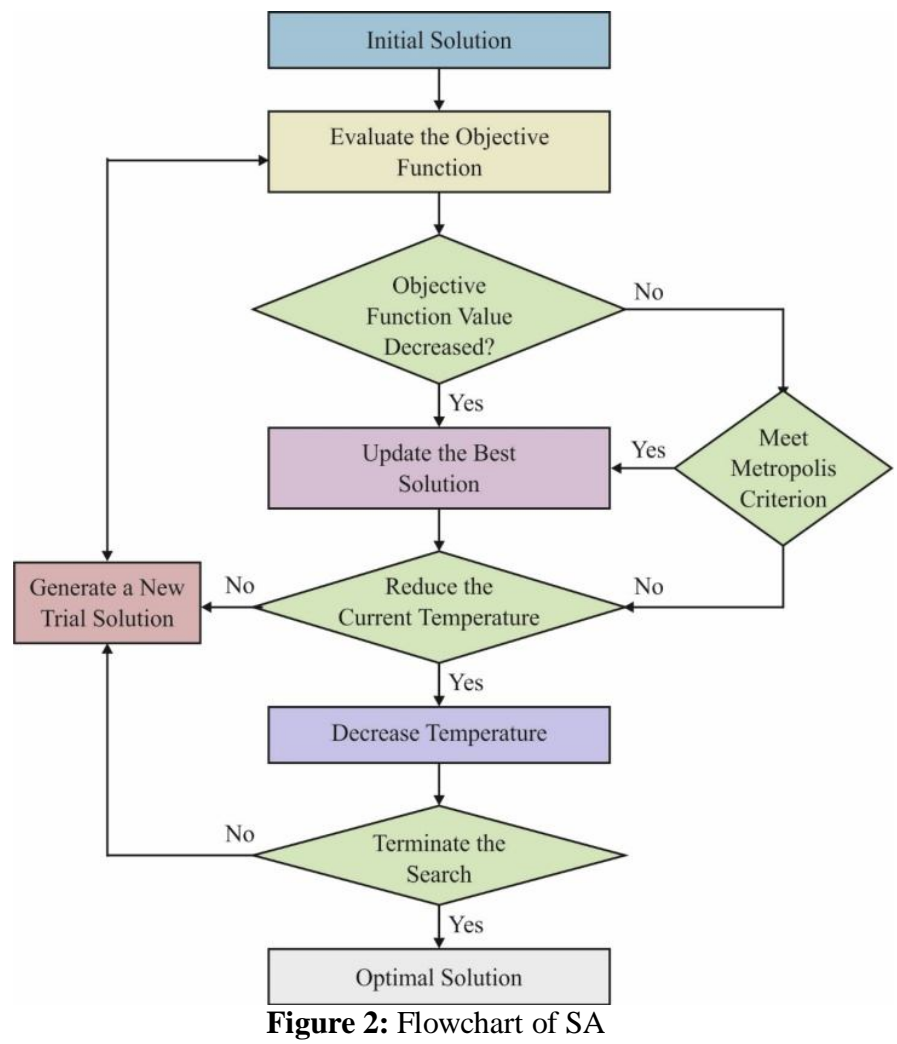

For maximizing the possibility of identifying states of lower energy at each value of $T$, thermal equilibrium should be reached. In order to achieve this, based on the Metropolis, an SA model has been developed for eliminating termination state from local minimum. The SA technique is composed of Metropolis suggestion at temperature $T$ for definite amount of time. Here, $T$ is fixed at with initial value, by expending sufficient time and it is considered as approximate thermal equilibrium. Moreover, a minimum reduction of $T$ is carried out and the process is iterated till the system is assumed as frozen. When cooling schedule is well developed, the consequent state is assumed as a near $\square$ optimal solution. Therefore, the entire process is moderately slow, due to the thermal equilibrium at every temperature $T$. The processes involved in SA is shown in Figure 2.

\section{A. Process involved in SA-FS model}

Primarily, the initial solution was chosen in a random manner that is considered to be a best solution. Then, the cost of initial solution is processed under the application of cost function. If temperature $T$ is not applicable in satisfying the termination condition, then neighboring solution is decided and determines the cost. When a cost of novel selected neighboring solution is minimum compared to therecent optimal solution, the present optimal solution is substituted with newly chosen neighbor solution. While cost of neighboring solution is maximum compared to the present optimal solution, a random value $q$ is selected within $(0,1)$. Hence, replacement of best solution is approved when a random value $q$ is lower than $e^{\frac{\operatorname{Cost}\left(v_{n}\right)-\operatorname{Cost}\left(v_{b}\right)}{T}}$. Once the temperature $T$ is reduced (7), these processes are followed until $T$ meets the termination criteria. At the end of the process, an optimal set of features were chosen by SA.

\subsection{KELM based Classification Process}

In this study, KELM is applied as a classifier to identify the existence of epileptic seizures. The KELM receives the input as optimal feature subset from SA and are applied to the KELM to carry out the classification process.

\section{A. Kernel Extreme Learning Machine}

If the feature mappings of ELM are unknown for individuals, a kernel trick is performed andKELM has been developed [15]. Figure 3 demonstrated the architecture of KELM. The final outcome shows that the KELM is applicable to reach normal generalization function with rapid learning speed when compared to classical SVR. Under the application of Eq. (12), the final weights of ELM are determined and eliminate the iteration of Gradient Decent (GD). Regardless, the architecture of ELM, such as the size of hidden layer is a hyper $\square$ parameter which contains significant impact on learning function that is highly difficult to select a best value under certain learning platform. In addition, support vector regression (SVR), is an implication of kernel models, in which kernel tricks are used for inner product. In general, ELM and SVR are assumed to be the variants of single $\square$ hidden $\square$ layer FFNN. Consequently, developers have presented the associations among ELM and SVR. KELM applies an expression $\varphi(x)$ by replacing $h(x)$ to refer the hidden layer. Finally, kernel matrix of ELM is represented as: 
P. Suguna et al., International Journal of Advanced Trends in Computer Science and Engineering, 9(4), July - August 2020,6464 - 6470

$$
K_{E L M}\left(x, x^{\prime}\right)=\varphi(x) \cdot \varphi\left(x^{\prime}\right)
$$

The output function $f(x)$ of KELM is equated as:

$$
\begin{gathered}
f(X)=\varphi(X) H^{T}\left(\frac{I}{C}+H H^{T}\right)^{-1} t \\
=\left[\begin{array}{l}
K_{E L M}\left(x \cdot x_{1}\right) \\
\vdots \\
K_{E L M}\left(x \cdot x_{N}\right)
\end{array}\right]^{\mathrm{T}}\left(\frac{I}{C}+K_{E L M}\right)^{-1} t
\end{gathered}
$$

Where, $C$ refers the regularization coefficient. The unknown hidden layer mapping $\phi(\cdot)$ of KELM is same as SVR, and kernel $K_{E L M}(\cdot, \cdot)$ has to be allocated. Consequently, the infrastructure of ELM is not essential to be determined. It is considered to have training set $T=\left(x_{i}, t_{i}\right), i=1, \ldots, N$, where $x_{i} \in \mathbb{R}^{n}$, and $t_{i} \in$.

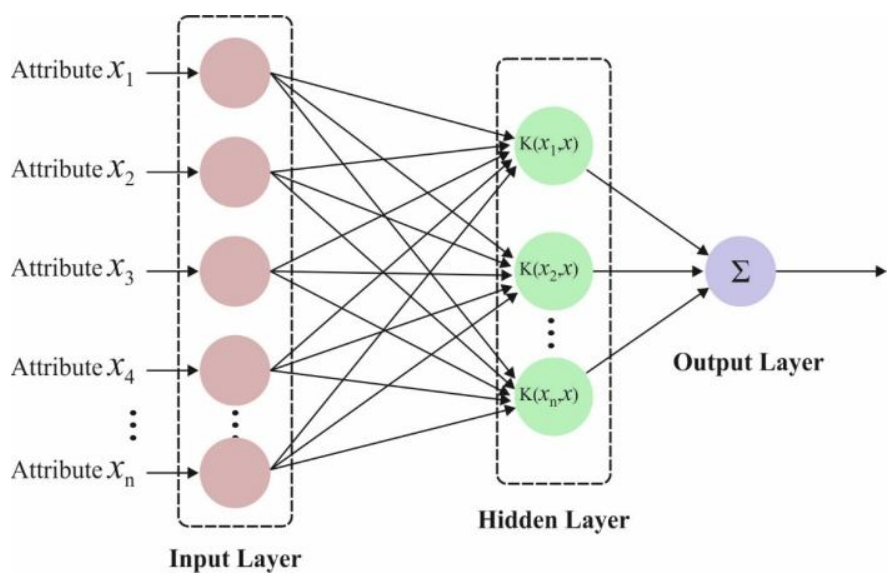

Figure 3: Architecture of KELM

The corresponding Lagrangian dual optimization issues of Eq. (5) is:

$$
L_{D}=\frac{1}{2}\|w\|^{2}+\frac{C}{2} \sum_{i=1}^{N} \xi_{i}^{2}-\sum_{i=1}^{N} \alpha_{i}\left(\varphi\left(x_{i}\right) w-t_{i}+\xi_{i}\right)
$$

where $\alpha_{j}$ implies the $i$ th Lagrangian multiplier. The optimality constraint of Eq. (5) is formalized as:

$$
\begin{gathered}
\frac{\partial L_{D}}{\partial w}=0 \rightarrow w=\sum_{i=1}^{N} \alpha_{i} \varphi\left(x_{i}\right)(6) \\
\frac{\partial L_{D}}{\partial \xi_{i}}=0 \rightarrow \alpha_{i}=C \xi_{i}, i=1, \ldots, N \\
\frac{\partial L_{D}}{\partial \alpha_{i}}=0 \rightarrow w^{T} \varphi\left(x_{i}\right)-t_{i}+\xi_{i}=0, i=1, \ldots, N
\end{gathered}
$$

By replacing Eqs. (6) and (7) into Eq. (8), it is obtained as,

$$
\alpha=\left(K_{E L M}+\frac{I}{C}\right)^{-1} t
$$

where $\quad=\left[\alpha_{1}, \ldots, \alpha_{N}\right]^{T}, K_{E L M(i, j)}=\varphi\left(x_{i}\right) \cdot \varphi\left(x_{j}\right) ; i, j=$ $1, \ldots, N$. Therefore, corresponding output function is:

$$
f\left(x^{\prime}\right)=K_{E L M}\left(x^{\prime}, x\right)\left(K_{E L M}+\frac{I}{C}\right)^{-1} t
$$

Eq. (5) is converted as follows:

$$
L_{D}=\alpha^{T} t-\frac{1}{2} \alpha^{T} K_{E L M} \alpha-\frac{1}{2 C} \alpha^{T} \alpha
$$

It is similar to kernel model; the type of kernel function as well as corresponding kernel parameters of KELM has to be determined without any theoretical guides.

\section{PERFORMANCE VALIDATION}

The proposed SA-KELM model has been simulated using Python 3.6.5 tool and the results are investigated under several measures. The parameter setting of SA-KELM model is given as follows. Maximum Number of Iterations (MaxIt) $=10, \quad$ Maximum Number of Sub-iterations (MaxSubIt) $=5$, initial temperature $T_{o}=10$, Temp. Reduction Rate (alpha) $=0.99$.

\subsection{Results analysis of FS process}

Figure4 displays the cost graph analysis of the presented SA-FS model on the applied dataset. The figure displayed that the SA-FS model has chosen an optimal set of features with least cost. It is also noticed that the cost gets decreased with an increase in number of iterations.

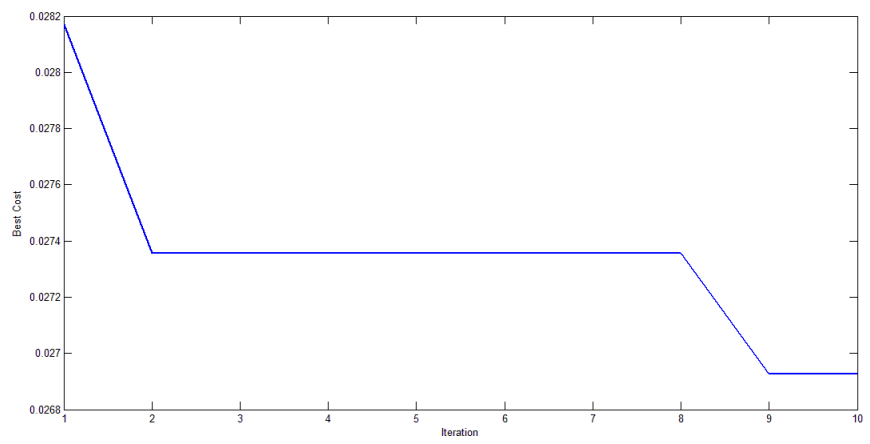

Figure 4: Cost Graph of SA-FS

Table 1 provides comparative results analysis of the SA-FS model with respect to number of chosen features and best cost. The table values signified that the GA-FS model has chosen a set of 141 features with the maximum best cost of 0.0378504. At the same time, the PSO-FS algorithm has reached to a selection of 134 features with the best cost of 0.0345691 . At last, the SA-FS model has attained a choice of 128 features with the minimum best cost of 0.026925 . These values notified that the SA-FS algorithm has attained maximum FS performance and outperforms the compared methods. Besides, Table 2 provides the list of 128 features chosen by the SA-FS model. 
Table 1 Result Analysis of Applied Feature Selection Methods

\begin{tabular}{|l|l|l|}
\hline Methods & Selected Features & Best Cost \\
\hline SA-FS & 128 & 0.026925 \\
\hline PSO-FS & 134 & 0.0345691 \\
\hline GA-FS & 141 & 0.0378504 \\
\hline
\end{tabular}

Table 2 Selected Features List

\begin{tabular}{|l|l|}
\hline $\begin{array}{l}\text { No. of } \\
\text { Selected } \\
\text { Feature } \\
\text { s }\end{array}$ & Features List \\
\hline & $121,6,159,165,127,59,106,79,142,68,48,177,108,151,168,35,99,81,143,76,136,3$ \\
& 1, \\
128 & $94,64,37,109,50,87,11,101,54,105,176,30,133,80,114,132,2,153,77,137,126,141$, \\
& $23,71,63,144,93,117,8,67,84,139,4,98,111,47,120,41,131,17,26,164,83,138,169$, \\
& $97,90,85,75,24,135,167,154,14,156,46,155,56,163,20,78,49,58,157,100,38,103$, \\
& $86,7,10,51,134,107,129,33,70,62,72,119,172,152,92,170,161,91,146,34,175,22$, \\
& $40,32,174,123,39,55,160,148,3,166,112,5,178,27,110,61,88$ \\
\hline
\end{tabular}

Figure 5 illustrates the confusion matrix generated by the KELM and SA-KELM models on the classification of binary classes. The figure depicted that the KELM model has classified a set of 1433 EEG signal instances as presence of seizure and $7828 \mathrm{EEG}$ signal instances as absence of seizure. On the other hand, it is observed that the SA-KELM model has classified a collection of 1498 EEG signal instances as presence of seizure and 7989 EEG signal instances as absence of seizure. These values indicated that the classification performance is improved with the inclusion of SA based FS process.

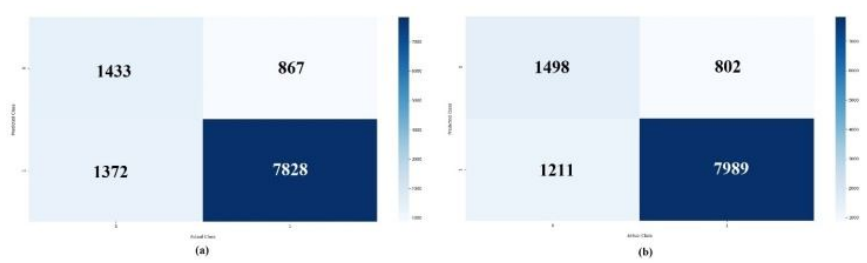

Figure 5: Confusion Matrix of Binary Class Dataset a) KELM b) SA-KELM
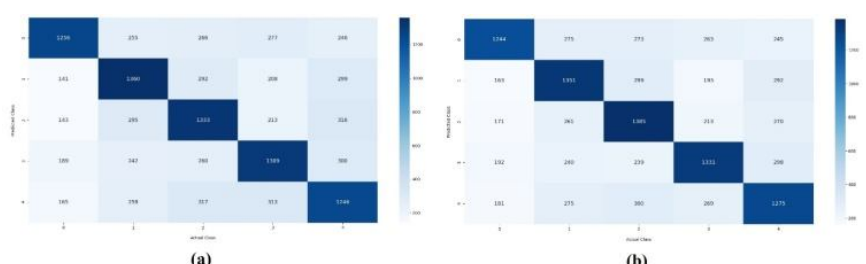

Figure 6:Confusion Matrix generation at the time of execution a) KELM b) SA-KELM

Figure6 depicts the confusion matrix created by the KELM and SA-KELM models on the classification of multiple classes at the time of execution. The confusion matrix is manipulated and is shown in Table 3. Figure7 represented that the KELM has classified a set of 1256, 1360, 1333, 1309 and 1246 EEG signal instances into positive class labels 0-4. Similarly, the SA-KELM model has classified a set of 8562 ,
8149, 8065, 8189 and 8039 EEG signal instances into negative class labels $0-4$. The figure also portrayed that the SA-KELM has classified a collection of 1244, 1351, 1385, 1331 and 1275 EEG signal instances into positive class labels 0-4. Likewise, the SA-KELM model has classified a set of 8562, 8149, 8065, 8189 and 8039 EEG signal instances into negative class labels $0-4$. In addition, the experimental screenshots generated at the time of execution of KELM and SA-KELM models are shown in Appendix I and II.
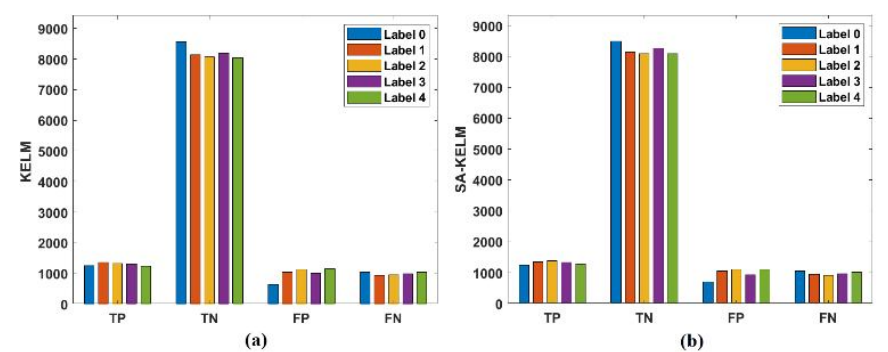

Figure7: Confusion Matrix of Multi-Class Dataset a) KELM b) SA-KELM

Table 3 Manipulation from Confusion Matrix of KELM and SA-KELM on Multi-Class Dataset

\begin{tabular}{|l|l|l|l|l|}
\hline \multirow{2}{*}{ Classes } & \multicolumn{4}{|l|}{ KELM } \\
\cline { 2 - 5 } & TP & TN & FP & FN \\
\hline Label 0 & 1256 & 8562 & 638 & 1044 \\
\hline Label 1 & 1360 & 8149 & 1051 & 940 \\
\hline Label 2 & 1333 & 8065 & 1135 & 967 \\
\hline Label 3 & 1309 & 8189 & 1011 & 991 \\
\hline Label 4 & 1246 & 8039 & 1161 & 1054 \\
\hline \multirow{2}{*}{ Classes } & SA-KELM & \multicolumn{3}{|l|}{} \\
\cline { 2 - 5 } & TP & TN & FP & FN \\
\hline Label 0 & 1244 & 8493 & 707 & 1056 \\
\hline Label 1 & 1351 & 8149 & 1051 & 949 \\
\hline Label 2 & 1385 & 8089 & 1111 & 915 \\
\hline Label 3 & 1331 & 8260 & 940 & 969 \\
\hline Label 4 & 1275 & 8095 & 1105 & 1025 \\
\hline
\end{tabular}

Table 4 and Figure 8 provides a comparison of the results offered by the KELM and SA-KELM models on the classification of binary and multiple classes interms of different measures. From the figure it is noted that the KELM has classified the EEG signals into binary class labels with the maximum sensitivity of $51.09 \%$, specificity of $90.03 \%$, accuracy of $80.53 \%$, F-score of $56.14 \%$, kappa of $43.79 \%$ and MCC of $44 \%$. At the same time, it is observed that the SA-KELM model has effectively classified the instances into binary classes with the maximum sensitivity of $51.09 \%$, specificity of $90.03 \%$, accuracy of $80.53 \%$, F-score of $56.14 \%$, kappa of $43.79 \%$ and MCC of $44 \%$. On the other 
hand, the KELM model has reached to a higher classifier results while classifying multiple classes with the sensitivity of $56.56 \%$, specificity of $89.14 \%$, accuracy of $82.62 \%$, F-score of $56.63 \%$, kappa of $45.78 \%$ and MCC of $45.88 \%$. Moreover, the SA-KELM model has demonstrated effective classification outcome with the sensitivity of $57.27 \%$, specificity of $89.32 \%$, accuracy of $82.91 \%$, F-score of $57.29 \%$, kappa of $46.62 \%$ and MCC of $46.69 \%$. These values showcased that the SA-KELM model under multi-classification has demonstrated superior results interms of distinct measures.

Table 4 Result Analysis of Proposed Method on Binary-Class and Multi-Class Dataset

\begin{tabular}{|c|c|c|c|c|c|c|}
\hline \multirow{2}{*}{ Methods } & Sensitivity & Specificity & Accuracy & F-score & Kappa & MCC \\
\hline & \multicolumn{6}{|c|}{ Binary Class Dataset } \\
\hline KELM & 51.09 & 90.03 & 80.53 & 56.14 & 43.79 & 44.00 \\
\hline \multirow[t]{2}{*}{ SA-KELM } & 55.30 & 90.88 & 82.49 & 59.81 & 48.72 & 48.99 \\
\hline & \multicolumn{6}{|c|}{ Multi-Class Dataset } \\
\hline KELM & 56.56 & 89.14 & 82.62 & 56.63 & 45.78 & 45.88 \\
\hline SA-KELM & 57.27 & 89.32 & 82.91 & 57.29 & 46.62 & 46.69 \\
\hline
\end{tabular}

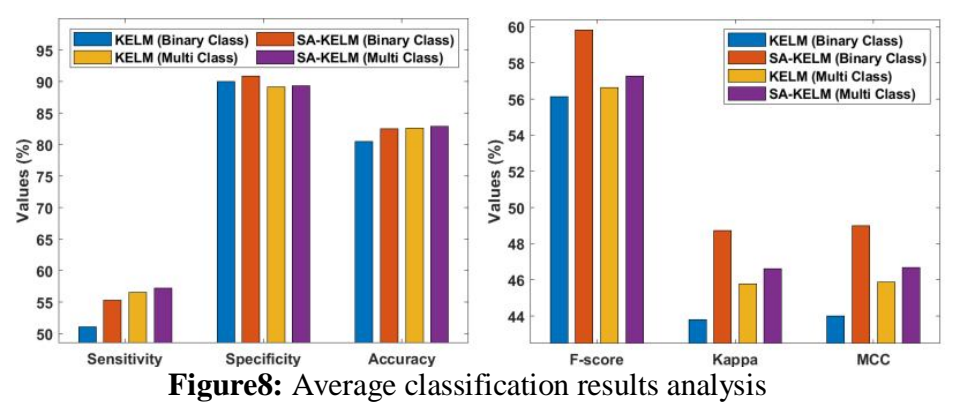

A detailed comparative results analysis of the proposed with existing models [16] interms of accuracy is made in Figure9. The figure showed that the KNN model has found to be an ineffective classifier which has attained a least accuracy of $76 \%$. On continuing with, the linear SVM and MLP models have obtained slightly higher accuracy values of $77.10 \%$ and $78 \%$. Followed by, the KELM (binary class) and M-Gaussian-SVM models has attained moderate accuracy of $80.53 \%$ and $81.70 \%$. Along with that, the Cubic-SVM model has reached to an accuracy of $82.30 \%$. The SA-KELM (binary class) model has shown better accuracy over the earlier methods with the accuracy of $82.49 \%$. Though the KELM (multi class) has exhibited near optimal classification with the accuracy of $82.62 \%$, the SA-KELM (multi-class) has resulted to a maximum classification accuracy of $82.91 \%$.

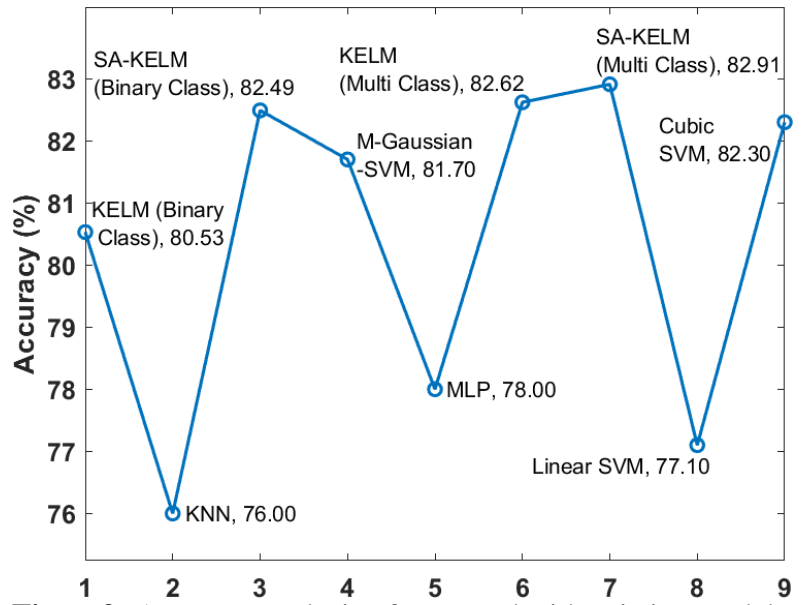

Figure9: Accuracy analysis of proposed with existing models

After observing the above mentioned tables and figures, it is evident that the SA-KELM model is found as an effective tool for epileptic seizure detection. It can be employed as a diagnosis tool to identify and classify different epileptic seizure class labels using EEG signals.

\section{CONCLUSION}

This paper has developed a new ML based epileptic seizure detection using SA-KELM model. At the initial stage, preprocessing is carried out on the EEG signals in two levels namely data normalization and class labeling. Next, SA is applied as a feature extractor to select an optimal set of features. Finally, KELM is employed as a classification model to detect the existence of different classes of epileptic seizure. The performance of the SA-KELM model is tested using EEG signal dataset and a detailed comparative analysis takes place to ensure the performance of the SA-KELM model. The performance of the SA-KELM model has been assessed using Epileptic Seizure Recognition dataset. The experimental results ensured the effectiveness of the SA-KELM under the classification of multiple classes with the maximum sensitivity of $57.27 \%$, specificity of $89.32 \%$, accuracy of $82.91 \%$, F-score of $57.29 \%$ and kappa of $46.62 \%$. In future, the performance of the epileptic seizure detection process is improved by the use of deep learning techniques.

\section{REFERENCES}

1. Ahmed. A. A. Gad-Elrab, Shereen A. El-aal, Neveen I. Ghali and Afaf A. S. Zaghrout, A Dynamic GeneticBased Context Modeling Approach in Internet of Things Environments, International Journal of Advanced Trends in Computer Science and Engineering, Volume 8 No. 6 (2019), pp. 2699 - 2709 https://doi.org/10.30534/ijatcse/2019/03862019

2. Bilal Hikmat Rasheed, M.Sivaram, D.Yuvaraj and A. Mohamed UvazeAhamed, An Improved Novel ANN Model for Detection of DDoS Attacks on Networks, International Journal of Advanced Trends in Computer Science and Engineering, vol. 8, no. 1, pp. 9-16, 2019. 
https://doi.org/10.30534/ijatcse/2019/0281.42019

3. Dinu A.J. and Ganesan R, Early detection of Alzheimer's disease using predictive k-NN instance based approach and T-Test Method, International Journal of Advanced Trends in Computer Science and Engineering, vol. 8, no. 1 , pp. $29-37,2019$. https://doi.org/10.30534/ijatcse/2019/0581.42019

4. El yadariAbdelbasset, Tridane Malika, Radid Mohamed and Belaaouad Said, Design of a Training Device Based on Integrative Alternation Teachers While Harnessing Artificial Intelligence, International Journal of Advanced Trends in Computer Science and Engineering, vol. 8, no. 1, pp. 261-266, 2019. https://doi.org/10.30534/ijatcse/2019/4081.42019

5. KocadagliOzan, Langari Reza. Classification of EEG signals for epileptic seizures using hybrid artificial neural networks based wavelet transforms and fuzzy relations. Expert SystAppl 2017;88:419-34.

6. Li Yang, Cui Wei-Gang, Huang Hui, Guo Yu-Zhu, Li Ke, Tan Tao. Epileptic seizure detection in EEG signals using sparse multiscale radial basis function networks and the Fisher vector approach. Knowl-Based Syst 2019;164:96-106.

7. Mutlu Ali Yener. Detection of epileptic dysfunctions in EEG signals using Hilbert vibration decomposition. Biomed Signal Process Control 2018;40:33-40.

8. Hassan AhnafRashik, SubasiAbdulhamit, Zhang Yanchun. Epilepsy seizure detection using complete ensemble empirical mode decomposition with adaptive noise. Knowl-Based Syst 2019:105333. https://doi.org/10.1016/j.knosys.2019.105333

9. Amin HafeezUllah, YusoffMohdZuki, Ahmad Rana Fayyaz. A novel approach based on wavelet analysis and arithmetic coding for automated detection and diagnosis of epileptic seizure in EEG signals using machine learning techniques. Biomed Signal Process Control 2020;56:101707.

10. BoonyakitanontPoomipat, Lek-uthaiApiwat, ChomthoKrisnachai, SongsiriJitkomut. A review of feature extraction and performance evaluation in epileptic seizure detection using EEG. Biomed Signal Process Control 2020;57:101702.

11. Bari MdFaizul, Fattah Shaikh Anowarul. Epileptic seizure detection in EEG signals using normalized IMFs in CEEMDAN domain and quadratic discriminant classifier. Biomed Signal Process Control 2020;58:101833.

12. Anupallavi S, MohanBabu G (2020) A novel approach based on BSPCI for quantifying functional connectivity pattern of the brain's region for the classifcationof epileptic seizure. J Ambient Intell Human Comput. https://doi.org/10.1007/s12652-020- 01774-w

13. Ogasawara, E., Martinez, L.C., De Oliveira, D., Zimbrão, G., Pappa, G.L. and Mattoso, M., 2010, July. Adaptive normalization: a novel data normalization approach for non-stationary time series. In The 2010 International Joint Conference on Neural Networks (IJCNN) (pp. 1-8). IEEE.

https://doi.org/10.1109/IJCNN.2010.5596746
14. Granville, V., Krivánek, M. and Rasson, J.P., 1994. Simulated annealing: A proof of convergence. IEEE transactions on pattern analysis and machine intelligence, 16(6), pp.652-656.

15. Lv, F. and Han, M., 2018. Hyperspectral image classification based on improved rotation forest algorithm. Sensors, 18(11), p.3601.

16. Guha, A., Ghosh, S., Roy, A. and Chatterjee, S., 2020. Epileptic Seizure Recognition Using Deep Neural Network. In Emerging Technology in Modelling and Graphics (pp. 21-28). Springer, Singapore. https://doi.org/10.1007/978-981-13-7403-6_3 